\title{
Evaluation of the My Diabetes Care Patient Portal Intervention: Protocol for a Pilot Randomized Controlled Trial
}

William Martinez, MD, MSc; Amber J Hackstadt, PhD; Gerald B Hickson, MD; S Trent Rosenbloom, MD, MPH; Tom A Elasy, MD, MPH

Department of Medicine, Vanderbilt University Medical Center, Nashville, TN, United States

Corresponding Author:

William Martinez, MD, MSc

Department of Medicine

Vanderbilt University Medical Center

2525 West End Avenue

Nashville, TN, 37203

United States

Phone: 16159361010

Fax: 16159361269

Email: william.martinez@vumc.org

\section{Abstract}

Background: My Diabetes Care (MDC) is a multi-faceted intervention embedded within an established patient portal, My Health at Vanderbilt. MDC is designed to help patients better understand their diabetes health data and support self-care. MDC uses infographics to visualize and summarize patients' diabetes health data, incorporates motivational strategies, provides literacy-level appropriate educational resources, and links to a diabetes online patient support community and diabetes news feeds.

Objective: This study aims to evaluate the effects of MDC on patient activation in adult patients with type 2 diabetes mellitus. Moreover, we plan to assess secondary outcomes, including system use and usability, and the effects of MDC on cognitive and behavioral outcomes (eg, self-care and self-efficacy).

Methods: We are conducting a 6-month, 2-arm, parallel-design, pragmatic pilot randomized controlled trial of the effect of MDC on patient activation. Adult patients with type 2 diabetes mellitus are recruited from primary care clinics affiliated with Vanderbilt University Medical Center. Participants are eligible for the study if they are currently being treated with at least one diabetes medication, are able to speak and read in English, are 21 years or older, and have an existing My Health at Vanderbilt account and reliable access to a desktop or laptop computer with internet access. We exclude patients living in long-term care facilities, patients with known cognitive deficits or severe visual impairment, and patients currently participating in any other diabetes-related research study. Participants are randomly assigned to MDC or usual care. We collect self-reported survey data, including the Patient Activation Measure (R) at baseline, 3 months, and 6 months. We will use mixed-effects regression models to estimate potentially time-varying intervention effects while adjusting for the baseline measure of the outcome. The mixed-effects model will use fixed effects for patient-level characteristics and random effects for health care provider variables (eg, primary care physicians).

Results: This study is ongoing. Recruitment was closed in May 2020; 270 patients were randomized. Of those randomized, most $(214 / 267,80.1 \%)$ were non-Hispanic White, and 13.1\% (35/267) were non-Hispanic Black, 43.7\% (118/270) reported being 65 years or older, and 33.6\% (90/268) reported limited health literacy. We obtained at least 95.6\% (258/270) completion among participants through the 3-month follow-up assessment.

Conclusions: This randomized controlled trial will be one of the first to evaluate a patient-facing diabetes digital health intervention delivered via a patient portal. By embedding MDC into Epic's MyChart platform with more than 127 million patient records, our intervention is directly integrated into routine care, highly scalable, and sustainable. Our findings and evolving patient portal functionality will inform the continued development of MDC to best meet users' needs and a larger trial focused on the impact of MDC on clinical end points.

Trial Registration: ClinicalTrials.gov NCT03947333; https://clinicaltrials.gov/ct2/show/NCT03947333

International Registered Report Identifier (IRRID): DERR1-10.2196/25955 
(JMIR Res Protoc 2021;10(5):e25955) doi: 10.2196/25955

\section{KEYWORDS}

patient portals; self-management; patient activation; diabetes mellitus; type 2; health literacy; health knowledge; attitudes; practice; mobile phone

\section{Introduction}

\section{Background}

Diabetes is a leading cause of several highly morbid and costly conditions, including chronic kidney disease, cardiovascular disease, and visual impairment [1]. Attention to diabetes self-management behaviors can help patients avoid or delay many diabetes-related complications; however, consistent engagement in self-care behaviors is challenging for many patients [1,2]. Patient activation (ie, knowledge, skills, and confidence in managing one's own health) is vital to achieving optimal diabetes self-management and is associated with lower health care costs [3-5].

Patient portals are computerized tools that can connect patients with electronic health data maintained by their health care system. Patient portals can provide an engaging and convenient means for patients to track and visualize health data, obtain education and guidance, and connect patients and doctors [6]. Research has shown that patient portals offer a promising platform to increase patient activation, enhance care, and promote self-management while overcoming the limitations of costly and difficult-to-scale face-to-face interventions [7,8]. We recently applied a user-centered design sprint methodology and key strategies for patient engagement to develop a patient portal intervention called My Diabetes Care (MDC; formerly Diabetes Dashboard) [9].

MDC is embedded within an established patient portal, My Health at Vanderbilt (MHAV), at Vanderbilt University Medical Center (VUMC) [10]. MDC is a multi-faceted intervention designed to help patients better understand their diabetes health data and support self-management [9]. MDC uses infographics to visualize and summarize patients' diabetes health data; incorporates motivational strategies (eg, social comparisons); provides literacy-level appropriate educational resources; contains secure messaging capability; and links to a diabetes online patient support community and diabetes news feeds, highlighting new discoveries, medicines, and recipes. MDC was founded on the well-established Chronic Care Model adapted for eHealth-eHealth Enhanced Chronic Care Model (eCCM) [11]. By leveraging elements within the model's 5 domains (self-management support, delivery system design, decision support, clinical information systems, and eHealth education), MDC has the potential to create more informed and activated patients, leading to improved outcomes (Figure 1).

Figure 1. The eHealth Enhanced Chronic Care Model with key aspects of My Diabetes Care shown in italics under the corresponding domain.

\section{The eHealth Enhanced Chronic Care Model}

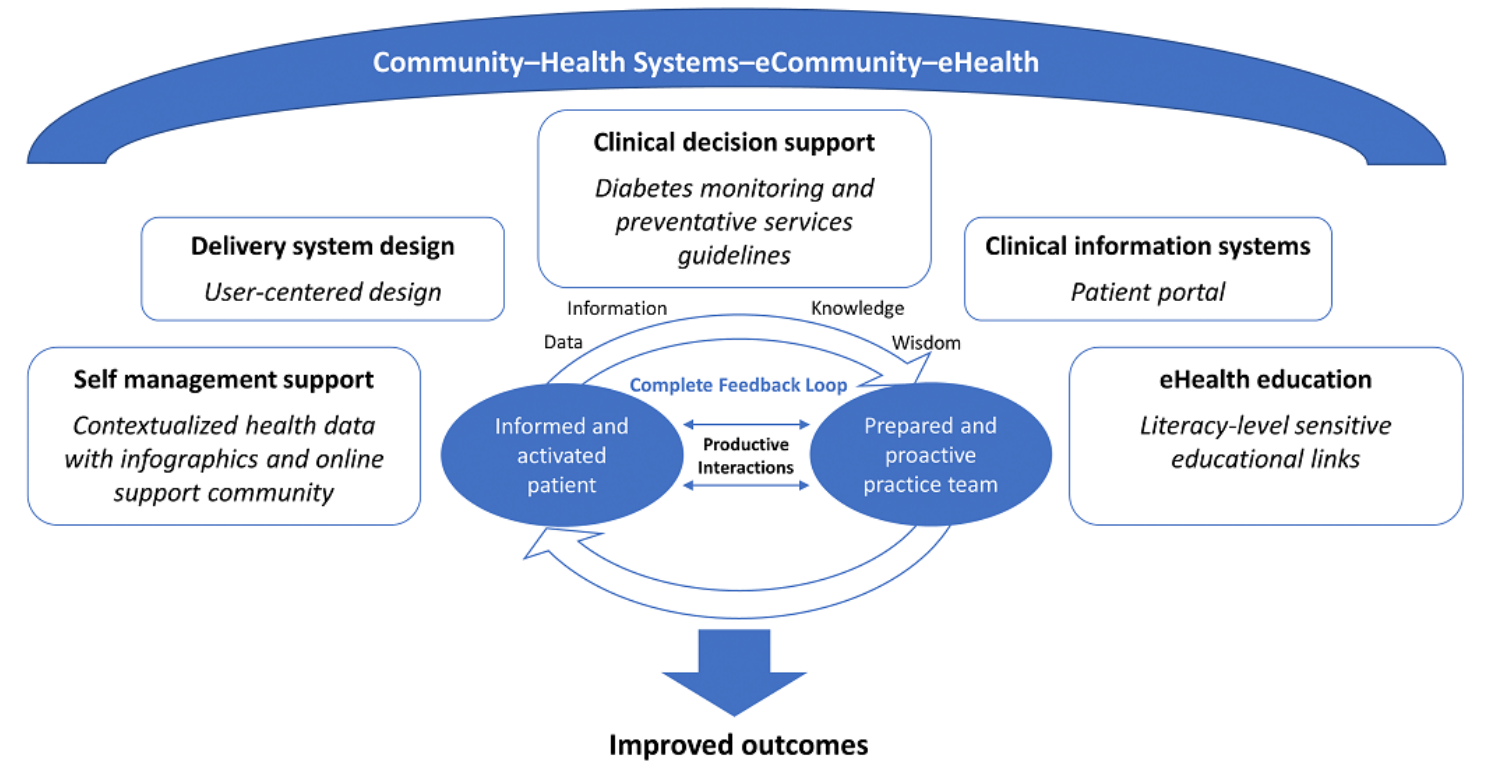

A 1-month usability study of MDC among 60 patients found that participants, including those with limited health literacy, highly rated MDC's usability and acceptability [12]. User experience data showed that most participants found that the infographics and links to literacy-level appropriate diabetes health information helped them better understand their diabetes health data. Participant feedback also identified areas for improvement, including adding information about diabetes medications and links to diabetes news feeds, highlighting new discoveries, medicines, and recipes. Consistent with the eCCM, the study also found a significant pre-post increase in patient activation scores among the study participants. However, a larger randomized controlled trial (RCT) is needed to assess MDC's impact on patient activation more definitively. 


\section{Objectives}

This study aims to evaluate the effects of MDC on patient activation in adult patients with type 2 diabetes mellitus (T2DM). In addition, we plan to explore secondary outcomes, including system use and usability, and the effects of MDC on diabetes self-efficacy, knowledge, self-care, medication adherence, distress, and clinical endpoints. The study will serve as a pilot for a larger definitive trial evaluating the effect of MDC on clinical endpoints.

\section{Methods}

\section{Study Design}

To achieve this objective, we are conducting a 6-month, 2-arm, parallel-design, pragmatic pilot RCT of MDC. Participants in both arms are told the purpose of the study is to determine satisfaction with 2 versions of MHAV among patients with diabetes. One version is the currently available version of MHAV. The second version of MHAV contains the MDC intervention. Participants in both arms complete the study questionnaires at 3 -time points: $\mathrm{T}_{0}=$ baseline, $\mathrm{T}_{1}=3$ months, and $\mathrm{T}_{2}=6$ months.

The study protocol is registered with ClinicalTrials.gov (ID NCT03947333) and is being conducted in accordance with the principles outlined in the CONSORT (Consolidated Standards of Reporting Trials) Statement, extension for pragmatic trials [13]. Pragmatic trials are designed to evaluate the real-world effectiveness of interventions in routine practice environments [14]. Unlike a strictly controlled trial, participants in our study are not constrained to receive a controlled dose of the intervention [14]. Due to necessity, participants are not blinded to the intervention, and we do not attempt to control participants' communication or information-seeking behaviors beyond the 2-arm randomization described here. The Vanderbilt University institutional review board approved this study.

\section{Recruitment and Eligibility}

Participants are recruited from 14 VUMC-affiliated adult primary care clinics located throughout Middle Tennessee (4 urban and 10 suburban clinics). An electronic health record (EHR; Epic Systems Corp) stores all clinical data. Patients receive access to their clinical data via an integrated and highly adopted patient portal, MHAV, that is accessible on desktops and via a native mobile app for iOS and Android mobile operating systems.

Participants are eligible for the study if they are a patient at a participating primary care clinic and have T2DM, are currently being treated with at least one diabetes medication, are able to speak and read in English, are 21 years or older, have an existing MHAV account, and have reliable access to a desktop or laptop computer with internet access. We exclude patients living in long-term care facilities, patients with known cognitive deficits, patients with a severe visual impairment, and patients currently participating in another diabetes-related research study.
On a rolling basis, potentially eligible patients are selected from a randomly ordered list of established adult patients with diabetes from participating clinic sites and are sent a recruitment letter describing the study. In addition, we also use My Research at Vanderbilt to send the recruitment letter to current patient portal users who elected to allow investigators to contact them about research opportunities via email. Interested patients contact a research assistant to learn more about the study. To enroll, participants complete a web-based study eligibility screener and electronic consent form on the web via REDCap (Research Electronic Data Capture) version 5.0.8. [15].

\section{Procedures and Randomization}

A study coordinator contacts all enrolled participants to review study procedures, answer the remaining questions, and confirm eligibility criteria. The enrolled participants are sent a baseline questionnaire via REDCap. After receiving the completed baseline questionnaire, the study coordinator randomly assigns participants to 1 of 2 groups: (1) intervention or (2) usual care. The randomization sequence was generated by the research team biostatistician using a permuted block randomization scheme stratified by clinic site and participants' age group (65 years and older vs younger than 65 years) to obtain balance across treatment groups on key variables. The randomized assignment for eligible participants is accessible only to the study coordinator and biostatistician using the REDCap randomization module; the other investigators are blinded. Once a randomization assignment is finalized, participants in both arms receive an email with their treatment assignment and an explanation of how to navigate to features of MHAV specific to their treatment group. Participants are asked to reply to the email affirming that they can access MHAV and/or MDC in accordance with their group assignment. Monthly quality assurance checks are used to ensure that MDC is functioning correctly (eg, displaying data correctly) and to ensure the fidelity of the intervention.

A participant may withdraw from the study at any time by notifying the study team. In addition, participants are withdrawn from the study by the investigators if they do not complete the baseline questionnaire needed for randomization. If a participant is withdrawn from the study for any reason, they are notified, and a reason is provided.

\section{Intervention and Control}

Participants randomized to the intervention arm are provided access to a version of MHAV embedded with MDC, as described in the Introduction section. Participants randomized to the intervention are advised to view MDC on a desktop or laptop device because the present version of MDC is not mobile friendly. Figure 2 shows a screenshot of MDC and illustrates its features $[16,17]$. Participants randomized to the usual care arm have access to the currently available version of MHAV, which includes the ability for patients to review pertinent health data, review medical information about their conditions, and communicate with their health care team. 
Figure 2. My Diabetes Care screenshot and features. Stars across the top fill in when the patient's glycated hemoglobin, blood pressure, cholesterol, or flu vaccine status are within goal range (ie, a value in the green zone on the infographic for each measure). Info icons provide a brief literacy-level appropriate description of each measure. Infographics display health data relative to a goal (green), caution (yellow), and warning (red) ranges. Patients Like Me indicates the average value of similar patients (ie, Vanderbilt patients with diabetes of the same gender, age group, and insulin-use status), and hovering over the icon reveals this description to the patient. Me indicates the patient's value, and hovering over the icon displays historical values. Literacy-level appropriate educational materials (hyperlinks) are paired with each measure. Message Your Doctor allows patients to send a secure message to members of their health care team. Online patient support community allows users to navigate directly to the American Diabetes Association (ADA) support community; a separate ADA account (username and password) is required. News Feeds provide newly published diabetes-related content, including recipes, discoveries, and new medications. FAQ provides answers to frequently asked questions regarding site features and navigation.

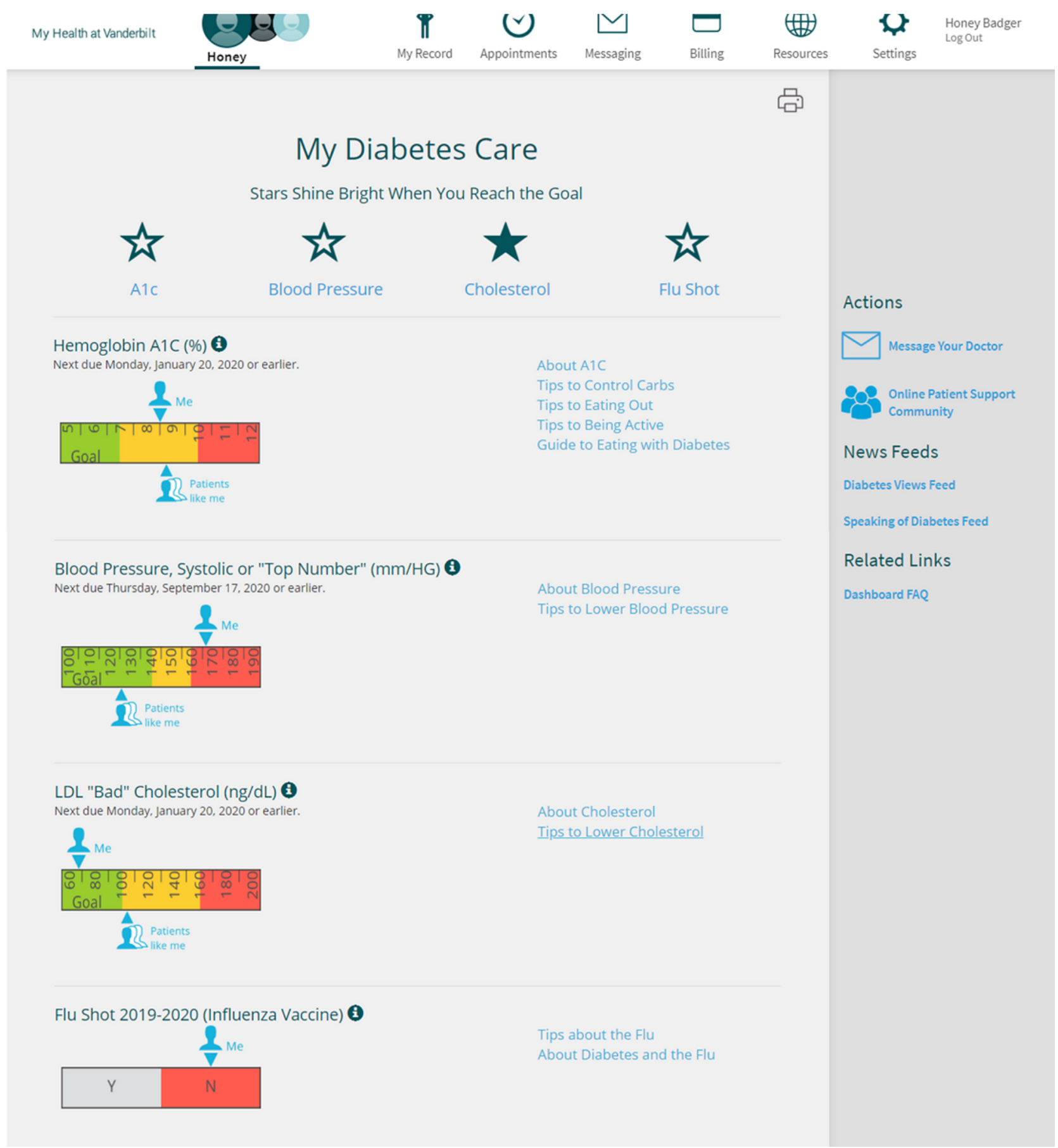

\section{Data Collection and Measures}

Enrolled participants receive 3 study questionnaires via REDCap at the associated time points $\left(\mathrm{T}_{0}-\mathrm{T}_{2}\right)$ : baseline questionnaire, 3-month follow-up questionnaire, and 6-month follow-up questionnaire. On the basis of pilot testing, we estimate the time to completion for the baseline questionnaire to be about 25

minutes and 20 minutes each for the 3-month and 6-month follow-up questionnaires. Participants are compensated US $\$ 40$ for completing the enrollment questionnaire and US \$35 each for completing the 3-month and 6-month follow-up questionnaires.

To describe the study population at baseline, we collect the following sociodemographic and clinical variables (Table 1). 
Health literacy is assessed by a validated 1-item screener asking respondents to rate their confidence independently filling out medical forms [18,19]. Consistent with previous studies, participants noting any lack of confidence are classified as having limited health literacy [20,21]. eHealth literacy is assessed by the 8-item eHealth Literacy Scale (eHEALS) [22]. The eHEALS uses a 5-point Likert scale ranging from strongly disagree to strongly agree. Total scores range from 8 (worst) to 40 (best). The presence of comorbidities (ie, hypertension and hyperlipidemia) is assessed by 2 clinicians who independently review patients' problem lists and medications abstracted from the EHR, and disagreements are resolved by consensus.

Table 2 shows the primary and secondary outcomes and related measures contained within the study questionnaires and their associated time points. The same study measures are administered to all participants in both arms, except for the system use and user experience, which contain items unique to the participants' assigned condition (ie, intervention vs control). 
Table 1. Sociodemographic and clinical variables collected at baseline.

\begin{tabular}{c} 
Variable an \\
\hline Age (years) \\
$<35$ \\
$35-44$ \\
$45-54$ \\
$55-64$ \\
$65-74$ \\
$75-84$ \\
$\geq 85$
\end{tabular}

Ethnicity

Questionnaire

Hispanic or Latino

Non-Hispanic or Latino

Race

Questionnaire

White

Black or African American

American Indian or Alaska Native

Asian

Native Hawaiian or Other Pacific Islander

More than one race

Other

Gender

Questionnaire

Female

Male

Other

Marital status

Questionnaire

Never married

Married or partnered

Separated or divorced

Widowed

\section{Education}

Questionnaire

8 grades or less

Some high school

High school graduate or GED ${ }^{\mathrm{a}}$

Some college or technical school

College graduate (bachelor's degree)

Some graduate work or school

Graduate degree

Employment status

Questionnaire

Working full-time: 35 hours or more a week

Working part-time: less than 35 hours a week

Unemployed or laid off and looking for work

Unemployed and not looking for work

Homemaker 
Variable and units or categories

Form of collection

In school

Retired

Disabled: not able to work

Something else

Insurance

Questionnaire

An individual plan: the member pays for the plan premium

A group plan through an employer or union: the employer pays all or part of the plan premium

US governmental health plan (eg, Military, CHAMPUS ${ }^{\mathrm{b}}$, Veterans Affairs, Medicaid, and Medicare)

I have not had an insurance plan in the past 12 months

Diabetes duration

Questionnaire

Years

\title{
Health literacy
}

Questionnaire

\author{
Adequate
}

Limited

eHealth literacy

Questionnaire

eHealth Literacy Scale score

Insulin status

Questionnaire

No

Yes

Previous diabetes self-management education

Questionnaire

No

Yes

Previous visit with dietician or nutritionist

Questionnaire

No

Yes

\section{Comorbidities}

EHR $^{\mathrm{c}}$ abstraction

Hyperlipidemia: no or yes

Hypertension: no or yes

Baseline clinical data

EHR abstraction

Glycated hemoglobin (\%)

Systolic blood pressure $(\mathrm{mm} \mathrm{Hg})$

Diastolic blood pressure $(\mathrm{mm} \mathrm{Hg})$

Mean arterial pressure $(\mathrm{mm} \mathrm{Hg})$

Low-density lipoprotein (mg/dL)

2019-2020 influenza vaccination status: no or yes

${ }^{\mathrm{a}}$ GED: Graduate Equivalency Degree.

${ }^{\mathrm{b}}$ CHAMPUS: Civilian Health and Medical Program of the Uniformed Services.

${ }^{\mathrm{c}}$ EHR: electronic health record. 
Table 2. Outcome measures.

\begin{tabular}{|c|c|c|c|c|}
\hline Outcomes & Measures & Variable type & Form of collection & Time points \\
\hline \multicolumn{5}{|l|}{ Primary outcome } \\
\hline Patient activation & Patient Activation Measure-13 (R) [23] & Continuous & Questionnaire & $\mathrm{T}_{0}{ }^{\mathrm{a}}, \mathrm{T}_{1}{ }^{\mathrm{b}}$, and $\mathrm{T}_{2}^{\mathrm{c}}$ \\
\hline \multicolumn{5}{|c|}{ Secondary cognitive and behavioral outcomes } \\
\hline Diabetes self-efficacy & Perceived Diabetes Self-Management Scale [24] & Continuous & Questionnaire & $\mathrm{T}_{0}, \mathrm{~T}_{1}$, and $\mathrm{T}_{2}$ \\
\hline Diabetes knowledge & Short Diabetes Knowledge Instrument [25] & Continuous & Questionnaire & $\mathrm{T}_{0}, \mathrm{~T}_{1}$, and $\mathrm{T}_{2}$ \\
\hline Diabetes self-care & Summary of Diabetes Self-Care Activities [26] & Continuous & Questionnaire & $\mathrm{T}_{0}, \mathrm{~T}_{1}$, and $\mathrm{T}_{2}$ \\
\hline $\begin{array}{l}\text { Diabetes medication } \\
\text { adherence }\end{array}$ & Adherence to Refills and Medications Scale for Diabetes [27] & Continuous & Questionnaire & $\mathrm{T}_{0}, \mathrm{~T}_{1}$, and $\mathrm{T}_{2}$ \\
\hline Diabetes distress & Problem Areas in Diabetes Scale-5 [28] & Continuous & Questionnaire & $\mathrm{T}_{0}, \mathrm{~T}_{1}$, and $\mathrm{T}_{2}$ \\
\hline $\begin{array}{l}\text { Understanding of dia- } \\
\text { betes health measures }\end{array}$ & $\begin{array}{l}\text { Unique study-specific items to assess participants' understanding } \\
\text { of measures of diabetes health status }\end{array}$ & Categorical & Questionnaire & $\mathrm{T}_{0}, \mathrm{~T}_{1}$, and $\mathrm{T}_{2}$ \\
\hline $\begin{array}{l}\text { Usability and satisfac- } \\
\text { tion }\end{array}$ & System usability scale [29] & Continuous & Questionnaire & $\mathrm{T}_{0}, \mathrm{~T}_{1}$, and $\mathrm{T}_{2}$ \\
\hline System use data & $\begin{array}{l}\text { - } \quad \text { Number of MHAV } \text { or MDC }^{\mathrm{e}} \text { visits } \\
\text { - } \quad \text { Nuration of MHAV or MDC visits } \\
\text { view most recent low-density lipoprotein value) } \\
\text { - } \quad \begin{array}{l}\text { Number of MDC information-seeking tasks performed } \\
\text { (eg, click links to embedded educational materials) }\end{array} \\
\text { - } \quad \begin{array}{l}\text { Number of MHAV or MDC health management-related } \\
\text { tasks performed (eg, utilization of embedded functionality }\end{array} \\
\text { to secure message health care team) } \\
\text { Number of MDC social support seeking tasks performed } \\
\text { (eg, click link to American Diabetes Association Online } \\
\text { Community) }\end{array}$ & Continuous & $\begin{array}{l}\text { System analytics } \\
\text { (if available), self- } \\
\text { report }\end{array}$ & $\mathrm{T}_{2}$ \\
\hline User experience & $\begin{array}{l}\text { Unique study-specific items to assess participants' perspectives } \\
\text { on specific features and functionality }\end{array}$ & $\begin{array}{l}\text { Categorical } \\
\text { and qualitative }\end{array}$ & Questionnaire & $\mathrm{T}_{2}$ \\
\hline Clinical endpoints & $\begin{array}{l}\text { Change in: } \\
\text { - } \quad \text { Glycated hemoglobin } \\
\text { - } \quad \text { Blood pressure } \\
\text { - } \quad \text { Low-density lipoproteins } \\
\text { - } \quad \text { Flu vaccination status }\end{array}$ & Continuous & $\mathrm{EHR}^{\mathrm{f}}$ abstraction & $\mathrm{T}_{0}, \mathrm{~T}_{1}$, and $\mathrm{T}_{2}$ \\
\hline
\end{tabular}

\footnotetext{
${ }^{\mathrm{a}} \mathrm{T}_{0}$ : baseline.

${ }^{\mathrm{b}_{\mathrm{T}}} \mathrm{T}_{1}$ : 3-month follow-up.

${ }^{\mathrm{c}} \mathrm{T}_{2}$ : 6-month follow-up.

${ }^{\mathrm{d}}$ MHAV: My Health at Vanderbilt.

${ }^{\mathrm{e}}$ MDC: My Diabetes Care.

${ }^{\mathrm{f}}$ EHR: electronic health record.
}

\section{Outcome Measures}

\section{Patient Activation}

The primary outcome measure is the change in patient activation as assessed by the Patient Activation Measure (R) (PAM) [23]. The 13-item PAM (R) survey uses a 4-point Likert scale of response options ranging from strongly disagree to strongly agree and has excellent internal consistency reliability (Cronbach $\alpha=.87$ ). The PAM-13 (R) survey item responses result in total raw scores ranging from 13 to 52, which are converted to the linear interval scale of patient activation scores, ranging from 0 (lowest activation) to 100 (highest activation).

\section{Diabetes Self-Efficacy}

The Perceived Diabetes Self-Management Scale (PDSMS) is used to measure diabetes self-efficacy (ie, how confident participants feel about their ability to perform multiple self-management tasks) [23]. The 8-item scale is scored on a 5-point Likert scale and has excellent internal consistency reliability (Cronbach $\alpha=.83$ ). The total PDSMS score ranges from 8 to 40, with higher scores indicating a greater confidence in managing diabetes. 


\section{Diabetes Knowledge}

The Short Diabetes Knowledge Instrument (SDKI) is used to measure diabetes knowledge, including diabetes diet, hypoglycemia symptoms, foot care, and the importance of physical activity [25]. The SDKI is a 13-item scale with scores ranging from 0 to 13, representing the number of items answered correctly, and has demonstrated good internal consistency reliability (Cronbach $\alpha=.73$ ) in a diverse sample of older adults.

\section{Diabetes Self-care}

Change in diabetes self-care is measured using the Summary of Diabetes Self-Care Activities (SDSCA) [26]. The SDSCA is an 11-item questionnaire of diabetes self-management that assesses the following 6 aspects of the diabetes self-care regimen: general diet ( 2 items), specific diet ( 2 items), exercise ( 2 items), blood glucose testing ( 2 items), foot care ( 2 items), and smoking (1 item). Item responses use the metric days per week, except for a single item about smoking status, which is a yes or no item. Each of the 6 aspects is assigned a mean score based on the number of days per week.

\section{Diabetes Medication Adherence}

Change in diabetes medication adherence is measured using the Adherence to Refills and Medications Scale-Diabetes (ARMS-D) [27]. The 11-item ARMS-D scale has excellent internal consistency reliability (Cronbach $\alpha=.86$ ). Responses range from $1=$ none of the time to $4=$ all of the time and are summed to generate an overall score ranging from 12 (best) to 48 (worst).

\section{Diabetes Distress}

The Problem Areas in Diabetes Scale (PAID-5) is used to measure changes in diabetes distress [28]. The 5-item unidimensional scale has scores ranging from 0 to 20 , with higher scores suggesting greater diabetes-related emotional distress. The PAID-5 has excellent internal consistency reliability (Cronbach $\alpha=.86$ ) and is associated with measures of depression.

\section{Understanding of Diabetes Health Measures}

Unique study-specific items are used to measure patients' understanding of the diabetes health measures displayed within MDC. For example, patients are asked to identify the goal range for glycated hemoglobin $\left(\mathrm{HbA}_{1 \mathrm{c}}\right)$, low-density lipoprotein (LDL) cholesterol, and systolic blood pressure.

\section{Satisfaction With Usability}

Usability of MDC is assessed by the 10-item System Usability Scale that measures users' perceptions of ease of use, the likability of the interface, and overall satisfaction using a 5-point Likert scale (strongly disagree to strongly agree) [29]. The item scores are summed and then converted to a score ranging from 0 (worst) to 100 (best), with a score above 68 considered above average [30].

\section{System Use Data}

We are collecting MDC system use data, including the total number of visits, total duration, and use of embedded educational resources; secure messaging; participation in the online patient support community; and hovers over the information icon about diabetes health measures and diabetes news feeds.

\section{User Experience}

User experience is assessed by unique study-specific multiple-choice and open-ended questions that solicit participants' perspectives on specific MDC features and functionality. For example, participants are asked to identify which features, if any, helped them better understand their diabetes health data and are asked to describe any problems they encountered using MDC.

\section{Clinical End Points}

Change in the following clinical endpoints is assessed by abstracting from the EHR the closest measurement on or before $\mathrm{T}_{0}, \mathrm{~T}_{1}$, and $\mathrm{T}_{2}$ time points for each of the following measures: $\mathrm{HbA}_{1 \mathrm{c}}$, blood pressure, LDL cholesterol, and flu vaccination status. For the final time point $\left(\mathrm{T}_{2}\right)$, we allow measures on or before $\mathrm{T}_{2}$ plus 2 weeks, as these measures can be reasonably assumed to reflect the study period.

\section{Data Analysis}

\section{Statistical Analysis Plan}

The study is designed to evaluate the effects of MDC on patient activation (primary analysis) and explore the effects on other secondary cognitive and behavioral outcomes relative to the control group. We will use mixed-effects regression models to estimate potentially time-varying intervention effects while adjusting for the baseline measure of the outcome. Nonlinear associations will be modeled with regression splines. The mixed-effects model will use fixed effects for patient-level characteristics and random effects for health care provider variables, such as primary care physicians. We will provide point estimates with CIs for each follow-up and graphically depict our results. The analysis will follow a conservative intention-to-treat principle, and participants with missing values will be included along with those with complete data. Multiple imputation will be used to impute the missing values. The analysis with multiple imputation assumes missing at random (ie, the model properly handles missing data by including covariates associated with reasons for dropout). The characteristics of participants who do not complete the study or do not comply with the treatment will be compared for both conditions. Mixed-effect models will also be used to evaluate the effects of MDC on secondary outcomes. For dichotomous secondary outcomes, such as flu vaccination status, we will use mixed-effects logistic regression. Given the smaller effective size when modeling dichotomous outcomes, the model for the dichotomous outcomes will not support as many covariates as the model for continuous outcomes.

\section{Primary Analysis}

We will test the impact of MDC on patient activation compared with the control condition (Table 2). We hypothesize that participants assigned to MDC will experience greater improvements in patient activation than participants assigned to the control condition. 


\section{Secondary Analysis}

In addition, we will test the effects of MDC on other behavioral and cognitive outcomes (Table 2). Finally, we will assess whether participants assigned to MDC experience greater improvements in $\mathrm{HbA}_{1 \mathrm{c}}$, blood pressure, LDL, and influenza vaccination status compared with those assigned to MHAV only.

\section{Sample Size and Power}

Assuming an up to a $20 \%$ dropout rate, approximately 240 patients (approximately 120 in each arm) are expected to complete the study. A conservative approach of a 2 -sided $t$ test performed at a 5\% significance level would detect an effect size of $0.36 \mathrm{SDs}$ for each continuous outcome with $80 \%$ power. In the context of the primary outcome PAM (R) survey and assuming a common SD of 12 points, this would be equivalent to detecting a true mean difference of 4.4 points; 4 -point changes in the PAM $(\mathrm{R})$ are associated with positive changes concerning particular diabetes self-care behaviors [31].

\section{Results}

\section{Recruitment}

Figure 3 shows the flowchart of the recruitment process. Recruitment began in March 2020 and ended in May 2020. Throughout the recruitment period, 4388 unique letters were sent to patients identified as potentially eligible. Separately, 2609 unique emails were sent to patients who use MHAV and previously agreed to be contacted by email about research studies for which they might be eligible. As it was not possible for the study team to cross reference the list of those who were sent letters against the list of those who were sent emails, some overlap is possible. The letters and emails generated 702 visits to the web-based REDCap eligibility screener, resulting in 576 completed screeners. Of the 576 complete screeners, 163 (28.3\%) were ineligible and $413(71.7 \%)$ were eligible. Of the 413 eligible screeners, 113 (27.4\%) declined to participate and $300(72.6 \%)$ were enrolled. We administratively withdrew $10 \%$ (30/300) of those enrolled, and the remaining 270 participants were randomized. 
Figure 3. Recruitment and enrollment flowchart. MHAV: My Health At Vanderbilt; MRAV: My Research At Vanderbilt; T2DM: type 2 diabetes mellitus.
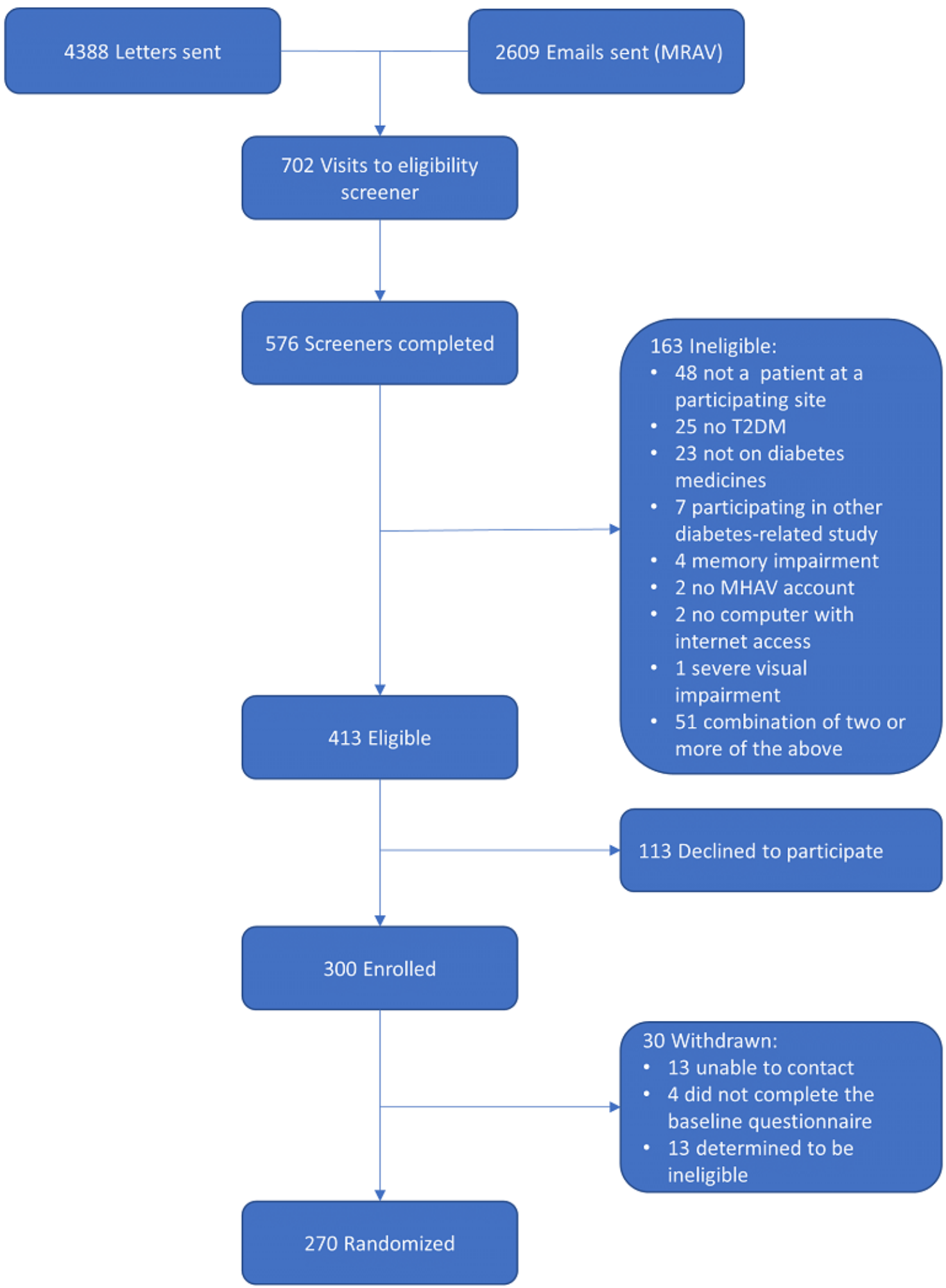

\section{Participants}

Of those randomized, most $(214 / 267,80.1 \%)$ were non-Hispanic White; $13.1 \%$ (35/267) were non-Hispanic Black; and 6.7\% (18/267) reported being of another race, including American Indian or Alaska Native, Asian, Native Hawaiian or other Pacific Islander, more than one race, Hispanic Black, and Hispanic White. In addition, 43.7\% (118/270) reported being 65 years or older. Furthermore, $10.1 \%(27 / 268)$ reported educational attainment of a high school degree or less, 33.6\% (90/268) had limited health literacy, and $39.6 \%(106 / 268)$ had only a US governmental health plan (eg, Military, Civilian Health and Medical Program of the Uniformed Services, Veterans Affairs, Medicaid, and Medicare). Approximately one-third (82/270, $30.4 \%$ ) were taking insulin, the mean duration of diabetes was 12.5 (SD 8.6) years, and the mean $\mathrm{HbA}_{1 \mathrm{c}}$ level at baseline was 
7.1 (SD 1.3). The overall clinical population with diabetes has a somewhat different demographic distribution: $66.49 \%$ (5317/7997) are non-Hispanic White, 21.83\%\% (1754/7997) are non-Hispanic Black, and 42.34\% (3386/7997) are 65 years or older. The mean $\mathrm{HbA}_{1 \mathrm{c}}$ of the 7997 patients within the overall clinical population and a laboratory value in the past 12 months was 7.40. As of October 2020, we have at least 95.6\% (258/270) completion among participants through the 3-month follow-up assessment.

\section{Discussion}

\section{Principal Findings}

Our study will be one of the very few RCTs to evaluate a patient-facing diabetes digital health intervention delivered via a patient portal. Although diabetes digital health interventions have great potential, their impact has been limited due to the difficulty in integrating the interventions into routine care [32]. By embedding MDC into Epic's MyChart platform with more than 127 million patient health records [33], our intervention is directly integrated into patients' health care systems and is highly scalable and sustainable. Unlike independent health apps, patient portals, by their very nature, are integrated into routine care and therefore offer greater potential for uptake and sustained use [7]. The challenge for health systems and investigators is how to make the most of patient portals to improve care.

We designed MDC to enhance and expand on existing aspects of patient portals, including access to personal health data and education, to better support diabetes self-management. Although patient portals offer easy access to personal health data, previous research suggests that complex data displays-showing many tests in small format on a single page without any indication of their clinical significance-make it difficult for patients to find and correctly interpret a particular test result [7]. MDC uses a simplified infographic to indicate normal, modestly abnormal, and more severely abnormal results and literacy-level appropriate materials to help patients better understand their diabetes health data. Thus, this research will inform how different data displays and user interface designs impact patients' ability to understand their personal health data.

Studies of other technology-enabled diabetes self-management solutions suggest additional strategies that may benefit patients $[32,34]$. These include analysis of patient-generated health data and tailored education and feedback [32,34]. To maximize scalability and sustainability, functionality enabling these strategies is best built directly into the EHR vendor's patient portal platform (eg, Epic's My Chart). Solutions built external to the platform may be more challenging to integrate into routine practice and run the risk of quickly becoming out of date and requiring reprogramming when vendors release platform updates. For this reason, we did not include these strategies in our intervention. However, EHR vendor solutions are emerging and may allow us to incorporate these additional patient engagement strategies into future iterations of MDC [35].

We designed MDC to be usable by the greatest number of patients, including those with limited health literacy [12].
Limited health literacy is typically associated with worse outcomes among patients with diabetes and can be a barrier to patient portal use [36,37]. Previous research has shown that patients with limited health literacy struggle to use patient portals because of complex medical terminology and a lack of literacy-level appropriate health information [20,21]. Although patient portals have the potential to worsen health inequities by further advantaging well-educated patients with greater resources, if designed and implemented appropriately, patient portals also have the potential to lower health literacy demands by ensuring that patients are presented with the health information and resources in a format that is convenient and easy to navigate and understand [38].

Our study population has a somewhat smaller proportion of racial or ethnic minorities than the overall clinical population, suggesting that additional strategies may be needed to increase adoption among these groups. Digital navigators - trained staff or volunteers who assist patients in accessing and learning how to use technology to meet their needs-have been used to increase patient portal adoption among vulnerable populations [20,39]. Smartphone use is increasingly common across different socioeconomic and racial or ethnic backgrounds, and for patients that lack broadband home internet connections, smartphones may be their only way to access the internet [40]. Thus, developing interventions suitable for mobile platforms may reduce barriers to adoption. Since the initiation of this trial, we have begun the development of a mobile-friendly version MDC that we hope will further increase its utility and accessibility.

Finally, given that racial and ethnic minorities are disproportionately affected by T2DM, future studies of MDC and other technology-delivered diabetes self-care interventions should consider using oversampling techniques, as demonstrated by Nelson et al $[41,42]$, to recruit study populations that closely represent the overall population of patients with T2DM. Doing so will help ensure that technology-delivered diabetes self-care interventions are effective in the populations with the greatest need and inform any revisions to those interventions and/or their implementation needed to address disparities.

\section{Limitations}

This study has important limitations. It relies on self-reported measures of patient activation and several secondary outcomes that are subject to social desirability and recall bias. However, the chosen measures are validated, widely used, and accepted, offering the advantage of being brief, inexpensive, and unobtrusive compared with more objective measures. Our study is powered to examine the effects of MDC on patient activation; therefore, analyses examining the effects of other outcomes (eg, self-care behaviors and $\mathrm{HbA}_{1 \mathrm{c}}$ ) and comparing the effects among subgroups (eg, patients with limited health literacy or poorly controlled diabetes) may be very informative but may also be underpowered. We hope that this study will serve as a pilot for a larger definitive trial evaluating the effect of MDC on clinical endpoints. Should MDC prove effective at increasing patient activation, the 6-month trial duration will not allow us to determine if the effect is temporary or sustained. A longer trial of a year or more in duration is needed to examine sustained effects. MDC is currently available only in English. This was 
necessary to increase the feasibility of designing the intervention and successfully completing this initial trial. However, diabetes disproportionately affects Spanish-speaking groups, so translation into Spanish will be an important goal, if MDC should prove beneficial. Finally, although patient portal interventions offer the advantages of direct integration into routine care, scalability, and sustainability, they are subject to inequities in patient portal adoption [43] and may appeal to more activated patients [44]. However, research shows that patient portal adoption is increasing [10,45], and if designed appropriately, patient portals could reduce health disparities $[38,46]$. Moreover, recent research finds that patient portal users have similar levels of patient activation as nonusers, although portal users are more likely to have internet access and a higher level of education [47].

\section{Conclusions}

We expect that this study will help determine the effectiveness of MDC in increasing patient activation among patients with diabetes. Beyond this primary objective, we will also be able to examine data on secondary cognitive, behavioral, and clinical outcomes and users' perceptions of and satisfaction with the intervention. Our findings and evolving patient portal functionality will inform the continued development of the intervention to best meet users' needs and a larger trial focused on the impact of MDC on clinical endpoints.

\section{Acknowledgments}

WM is the principal investigator who led the development of the research protocol and oversaw the execution of the research plan. WM and AJH wrote the manuscript. All coauthors are coinvestigators of the project, contributed to developing the study protocol, and read and edited the manuscript. This research is funded by the National Institutes of Health's National Institute of Diabetes and Digestive and Kidney (grants K23 DK106511 and P30DK092986) and National Institutes of Health's National Center for Advancing Translational Sciences (grant UL1TR000445). The authors thank Vanderbilt Adult Primary Care, Vanderbilt Health IT, and the study participants for their contributions to this research.

\section{Conflicts of Interest}

None declared.

\section{References}

1. Centers for Disease Control and Prevention. National diabetes statistics report. Atlanta, GA: Centers for Disease Control and Prevention, US Department of Health and Human Services; 2020. URL: https://www.cdc.gov/diabetes/pdfs/data/ statistics/national-diabetes-statistics-report.pdf

2. Powers MA, Bardsley J, Cypress M, Duker P, Funnell MM, Fischl AH, et al. Diabetes Self-management Education and Support in Type 2 Diabetes. Diabetes Educ 2017 Feb;43(1):40-53. [doi: 10.1177/0145721716689694] [Medline: 28118121]

3. Hibbard JH, Greene J. What the evidence shows about patient activation: better health outcomes and care experiences; fewer data on costs. Health Aff (Millwood) 2013 Feb;32(2):207-214. [doi: 10.1377/hlthaff.2012.1061] [Medline: 23381511]

4. Zimbudzi E, Lo C, Ranasinha S, Kerr PG, Polkinghorne KR, Teede H, et al. The association between patient activation and self-care practices: A cross-sectional study of an Australian population with comorbid diabetes and chronic kidney disease. Health Expect 2017 Dec;20(6):1375-1384 [FREE Full text] [doi: 10.1111/hex.12577] [Medline: 28675539]

5. Almutairi N, Hosseinzadeh H, Gopaldasani V. The effectiveness of patient activation intervention on type 2 diabetes mellitus glycemic control and self-management behaviors: A systematic review of RCTs. Prim Care Diabetes 2020 Feb;14(1):12-20. [doi: 10.1016/j.pcd.2019.08.009] [Medline: 31543458]

6. Tulu B, Trudel J, Strong DM, Johnson SA, Sundaresan D, Garber L. Patient Portals: An Underused Resource for Improving Patient Engagement. Chest 2016 Jan;149(1):272-277. [doi: 10.1378/chest.14-2559] [Medline: 26066707]

7. Baldwin J, Singh H, Sittig D, Giardina T. Patient portals and health apps: Pitfalls, promises, and what one might learn from the other. Healthc (Amst) 2017 Sep;5(3):81-85 [FREE Full text] [doi: 10.1016/j.hjdsi.2016.08.004] [Medline: 27720139]

8. Dalal AK, Dykes P, Samal L, McNally K, Mlaver E, Yoon CS, et al. Potential of an Electronic Health Record-Integrated Patient Portal for Improving Care Plan Concordance during Acute Care. Appl Clin Inform 2019 May 29;10(03):358-366. [doi: $10.1055 / \mathrm{s}-0039-1688831$ ] [Medline: $\underline{31141830}$ ]

9. Martinez W, Threatt AL, Rosenbloom ST, Wallston KA, Hickson GB, Elasy TA. A Patient-Facing Diabetes Dashboard Embedded in a Patient Web Portal: Design Sprint and Usability Testing. JMIR Hum Factors 2018 Sep 24;5(3):e26 [FREE Full text] [doi: 10.2196/humanfactors.9569] [Medline: 30249579]

10. Steitz B, Wong J, Cobb J, Carlson B, Smith G, Rosenbloom S. Policies and procedures governing patient portal use at an Academic Medical Center. JAMIA Open 2019 Dec;2(4):479-488 [FREE Full text] [doi: 10.1093/jamiaopen/ooz039] [Medline: 32025645]

11. Gee PM, Greenwood DA, Paterniti DA, Ward D, Miller LMS. The eHealth Enhanced Chronic Care Model: a theory derivation approach. J Med Internet Res 2015 Apr 01;17(4):e86 [FREE Full text] [doi: 10.2196/jmir.4067] [Medline: 25842005]

12. Martinez W, Hackstadt AJ, Hickson GB, Knoerl T, Rosenbloom ST, Wallston KA, et al. The My Diabetes Care Patient Portal Intervention: Usability and Pre-Post Assessment. Applied Clinical Informatics 2021 Forthcoming. [doi: 10.1055/s-0041-1730324] 
13. Zwarenstein M, Treweek S, Gagnier JJ, Altman DG, Tunis S, Haynes B, CONSORT group, Pragmatic Trials in Healthcare (Practihc) group. Improving the reporting of pragmatic trials: an extension of the CONSORT statement. BMJ 2008 Nov 11;337:a2390 [FREE Full text] [doi: 10.1136/bmj.a2390] [Medline: 19001484]

14. Ford I, Norrie J. Pragmatic Trials. N Engl J Med 2016 Aug 04;375(5):454-463. [doi: 10.1056/nejmra1510059] [Medline: 27518663]

15. Harris PA, Taylor R, Thielke R, Payne J, Gonzalez N, Conde JG. Research electronic data capture (REDCap)--a metadata-driven methodology and workflow process for providing translational research informatics support. J Biomed Inform 2009 Apr;42(2):377-381 [FREE Full text] [doi: 10.1016/j.jbi.2008.08.010] [Medline: 18929686]

16. Wolff K, Chambers L, Bumol S, White RO, Gregory BP, Davis D, et al. The PRIDE (Partnership to Improve Diabetes Education) Toolkit: Development and Evaluation of Novel Literacy and Culturally Sensitive Diabetes Education Materials. Diabetes Educ 2016 Feb;42(1):23-33 [FREE Full text] [doi: 10.1177/0145721715620019] [Medline: 26647414]

17. Learning About Diabetes. Learning About Diabetes Inc. URL: http://www.learningaboutdiabetes.org/ [accessed 2018-02-10] [WebCite Cache ID 6x8VV2atx]

18. Chew L, Bradley K, Boyko E. Brief questions to identify patients with inadequate health literacy. Fam Med 2004 Sep;36(8):588-594 [FREE Full text] [Medline: 15343421]

19. Sarkar U, Piette JD, Gonzales R, Lessler D, Chew LD, Reilly B, et al. Preferences for self-management support: findings from a survey of diabetes patients in safety-net health systems. Patient Educ Couns 2008 Jan;70(1):102-110 [FREE Full text] [doi: 10.1016/j.pec.2007.09.008] [Medline: 17997264]

20. Tieu L, Schillinger D, Sarkar U, Hoskote M, Hahn K, Ratanawongsa N, et al. Online patient websites for electronic health record access among vulnerable populations: portals to nowhere? J Am Med Inform Assoc 2017 Apr 01;24(e1):e47-e54 [FREE Full text] [doi: 10.1093/jamia/ocw098] [Medline: 27402138]

21. Tieu L, Sarkar U, Schillinger D, Ralston JD, Ratanawongsa N, Pasick R, et al. Barriers and Facilitators to Online Portal Use Among Patients and Caregivers in a Safety Net Health Care System: A Qualitative Study. J Med Internet Res 2015 Dec 03;17(12):e275 [FREE Full text] [doi: 10.2196/jmir.4847] [Medline: 26681155]

22. Norman CD, Skinner HA. eHEALS: The eHealth Literacy Scale. J Med Internet Res 2006 Nov 14;8(4):e27 [FREE Full text] [doi: $10.2196 / j m i r .8 .4 . e 27]$ [Medline: 17213046$]$

23. Hibbard JH, Mahoney ER, Stockard J, Tusler M. Development and testing of a short form of the patient activation measure. Health Serv Res 2005 Dec;40(6 Pt 1):1918-1930 [FREE Full text] [doi: 10.1111/j.1475-6773.2005.00438.x] [Medline: $\underline{16336556]}$

24. Wallston KA, Rothman RL, Cherrington A. Psychometric properties of the Perceived Diabetes Self-Management Scale (PDSMS). J Behav Med 2007 Oct;30(5):395-401. [doi: 10.1007/s10865-007-9110-y] [Medline: 17522972]

25. Quandt SA, Ip EH, Kirk JK, Saldana S, Chen S, Nguyen H, et al. Assessment of a short diabetes knowledge instrument for older and minority adults. Diabetes Educ 2014;40(1):68-76 [FREE Full text] [doi: 10.1177/0145721713508824] [Medline: 24163359]

26. Toobert DJ, Hampson SE, Glasgow RE. The summary of diabetes self-care activities measure: results from 7 studies and a revised scale. Diabetes Care 2000 Jul;23(7):943-950 [FREE Full text] [doi: 10.2337/diacare.23.7.943] [Medline: 10895844]

27. Mayberry LS, Gonzalez JS, Wallston KA, Kripalani S, Osborn CY. The ARMS-D out performs the SDSCA, but both are reliable, valid, and predict glycemic control. Diabetes Res Clin Pract 2013 Nov;102(2):96-104 [FREE Full text] [doi: 10.1016/j.diabres.2013.09.010] [Medline: 24209600]

28. McGuire BE, Morrison TG, Hermanns N, Skovlund S, Eldrup E, Gagliardino J, et al. Short-form measures of diabetes-related emotional distress: the Problem Areas in Diabetes Scale (PAID)-5 and PAID-1. Diabetologia 2010 Jan;53(1):66-69. [doi: 10.1007/s00125-009-1559-5] [Medline: 19841892]

29. Brooke J. SUS-A quick and dirty usability scale. In: Jordan PW, Thomas B, Weerdmeester BA, McClelland IL, editors. Usability evaluation in industry. London: Taylor and Francis; 1996:189-194.

30. Bangor A, Kortum PT, Miller JT. An Empirical Evaluation of the System Usability Scale. International Journal of Human-Computer Interaction 2008 Jul 30;24(6):574-594. [doi: 10.1080/10447310802205776]

31. Rask KJ, Ziemer DC, Kohler SA, Hawley JN, Arinde FJ, Barnes CS. Patient activation is associated with healthy behaviors and ease in managing diabetes in an indigent population. Diabetes Educ 2009;35(4):622-630. [doi: 10.1177/0145721709335004] [Medline: 19419972]

32. Nelson L, Williamson S, Nigg A, Martinez W. Implementation of Technology-Delivered Diabetes Self-care Interventions in Clinical Care: a Narrative Review. Curr Diab Rep 2020 Nov 18;20(12):71. [doi: 10.1007/s11892-020-01356-2] [Medline: 33206241]

33. Moukheiber Z. Behind Epic Systems, A Low-Key Health IT Company Called InterSystems. Forbes. 2013. URL: https:/ /www.forbes.com/sites/zinamoukheiber/2013/03/04/behind-epic-systems-a-low-key-health-it-company-called-intersystems/ ?sh=6d024fced88a [accessed 2019-04-13]

34. Greenwood DA, Gee PM, Fatkin KJ, Peeples M. A Systematic Review of Reviews Evaluating Technology-Enabled Diabetes Self-Management Education and Support. J Diabetes Sci Technol 2017 Sep;11(5):1015-1027. [doi:

10.1177/1932296817713506] [Medline: 28560898] 
35. Lewinski A, Drake C, Shaw R, Jackson G, Bosworth H, Oakes M, et al. Bridging the integration gap between patient-generated blood glucose data and electronic health records. J Am Med Inform Assoc 2019 Jul 01;26(7):667-672 [FREE Full text] [doi: 10.1093/jamia/ocz039] [Medline: $\underline{\text { 31192360] }}$

36. Marciano L, Camerini A, Schulz PJ. The Role of Health Literacy in Diabetes Knowledge, Self-Care, and Glycemic Control: a Meta-analysis. J Gen Intern Med 2019 Jun;34(6):1007-1017 [FREE Full text] [doi: 10.1007/s11606-019-04832-y] [Medline: $\underline{30877457]}$

37. Sarkar U, Karter AJ, Liu JY, Adler NE, Nguyen R, Lopez A, et al. The literacy divide: health literacy and the use of an internet-based patient portal in an integrated health system-results from the diabetes study of northern California (DISTANCE). J Health Commun 2010;15 Suppl 2:183-196 [FREE Full text] [doi: 10.1080/10810730.2010.499988] [Medline: 20845203]

38. Institute of Medicine Roundtable on Health Literacy. Health Literacy, eHealth, and Communication. Putting the Consumer First: Workshop Summary. Washington, DC: National Academies Press; 2009. URL: https://www.ncbi.nlm.nih.gov/books/ NBK36296/ [accessed 2020-04-13]

39. McAlearney AS, Sieck CJ, Hefner JL, Aldrich AM, Walker DM, Rizer MK, et al. High Touch and High Tech (HT2) Proposal: Transforming Patient Engagement Throughout the Continuum of Care by Engaging Patients with Portal Technology at the Bedside. JMIR Res Protoc 2016 Nov 29;5(4):e221 [FREE Full text] [doi: 10.2196/resprot.6355] [Medline: 27899338]

40. Anderson M. Mobile technology and home broadband 2019. Pew Research Center. 2019 Jun 13. URL: https://www. pewresearch.org/internet/2019/06/13/mobile-technology-and-home-broadband-2019/ [accessed 2020-04-13]

41. Nelson LA, Greevy RA, Spieker A, Wallston KA, Elasy TA, Kripalani S, et al. Effects of a Tailored Text Messaging Intervention Among Diverse Adults With Type 2 Diabetes: Evidence From the 15-Month REACH Randomized Controlled Trial. Diabetes Care 2021 Jan;44(1):26-34. [doi: 10.2337/dc20-0961] [Medline: 33154039]

42. Nelson LA, Wallston KA, Kripalani S, Greevy RA, Elasy TA, Bergner EM, et al. Mobile Phone Support for Diabetes Self-Care Among Diverse Adults: Protocol for a Three-Arm Randomized Controlled Trial. JMIR Res Protoc 2018 Apr 10;7(4):e92 [FREE Full text] [doi: 10.2196/resprot.9443] [Medline: 29636319]

43. Anthony DL, Campos-Castillo C, Lim PS. Who Isn't Using Patient Portals And Why? Evidence And Implications From A National Sample Of US Adults. Health Aff (Millwood) 2018 Dec;37(12):1948-1954. [doi: 10.1377/hlthaff.2018.05117] [Medline: $\underline{30633673}$ ]

44. Hibbard JH, Greene J. Who Are We Reaching Through the Patient Portal: Engaging the Already Engaged? International Journal of Person Centered Medicine 2011;1(4):788-793. [doi: 10.5750/ijpcm.v1i4.152]

45. Turner K, Hong Y, Yadav S, Huo J, Mainous A. Patient portal utilization: before and after stage 2 electronic health record meaningful use. J Am Med Inform Assoc 2019 Oct 01;26(10):960-967 [FREE Full text] [doi: 10.1093/jamia/ocz030] [Medline: $\underline{30947331]}$

46. Apter AJ. Can patient portals reduce health disparities? A perspective from asthma. Ann Am Thorac Soc 2014 May;11(4):608-612. [doi: 10.1513/AnnalsATS.201401-032PS] [Medline: 24640983]

47. Ancker JS, Osorio SN, Cheriff A, Cole CL, Silver M, Kaushal R. Patient activation and use of an electronic patient portal. Inform Health Soc Care 2015;40(3):254-266. [doi: 10.3109/17538157.2014.908200] [Medline: 24786648]

\author{
Abbreviations \\ ARMS-D: Adherence to Refills and Medications Scale-Diabetes \\ eCCM: eHealth Enhanced Chronic Care Model \\ eHEALS: eHealth Literacy Scale \\ EHR: electronic health record \\ $\mathbf{H b A}_{1 \mathbf{c}}$ : glycated hemoglobin \\ LDL: low-density lipoprotein \\ MDC: My Diabetes Care \\ MHAV: My Health at Vanderbilt \\ PAID-5: Problem Areas in Diabetes Scale \\ PAM (R): Patient Activation Measure (R) \\ PDSMS: Perceived Diabetes Self-Management Scale \\ RCT: randomized controlled trial \\ REDCap: Research Electronic Data Capture \\ SDKI: Short Diabetes Knowledge Instrument \\ SDSCA: Summary of Diabetes Self-Care Activities \\ T2DM: type 2 diabetes mellitus \\ VUMC: Vanderbilt University Medical Center
}


Edited by G Eysenbach; submitted 02.12.20; peer-reviewed by ML Nguyen, H Imeri; comments to author 29.01.21; revised version received 01.02.21; accepted 25.02.21; published 25.05.21

Please cite as:

Martinez, W, Hackstadt AJ, Hickson GB, Rosenbloom ST, Elasy TA

Evaluation of the My Diabetes Care Patient Portal Intervention: Protocol for a Pilot Randomized Controlled Trial JMIR Res Protoc 2021;10(5):e25955

URL: https://www.researchprotocols.org/2021/5/e25955

doi: $10.2196 / 25955$

PMID:

CWilliam Martinez, Amber J Hackstadt, Gerald B Hickson, S Trent Rosenbloom, Tom A Elasy. Originally published in JMIR Research Protocols (https://www.researchprotocols.org), 25.05.2021. This is an open-access article distributed under the terms of the Creative Commons Attribution License (https://creativecommons.org/licenses/by/4.0/), which permits unrestricted use, distribution, and reproduction in any medium, provided the original work, first published in JMIR Research Protocols, is properly cited. The complete bibliographic information, a link to the original publication on https://www.researchprotocols.org, as well as this copyright and license information must be included. 